\title{
THE FUSION OF EDGE DETECTION AND MATHEMATICAL MORPHOLOGY ALGORITHM FOR SHAPE BOUNDARY RECOGNITION
}

\author{
${ }^{1}$ Mahmod Othman, ${ }^{2}$ Sharifah Lailee Syed Abdullah, ${ }^{3}$ Khairul Adilah Ahmad, \\ ${ }^{2}$ Mohd. Nazari Abu Bakar \& ${ }^{3}$ Ab. Razak Mansor \\ ${ }^{1}$ Universiti Teknologi Petronas, Malaysia \\ ${ }^{2}$ Universiti Teknologi MARA, Perlis, Malaysia \\ ${ }^{3}$ Universiti Teknologi MARA, Kedah, Malaysia \\ mahmod.othman@petronas.com; shlailee@perlis.uitm.edu.my; \\ adilah475@kedah.uitm.edu.my; mohdnazari@perlis.uitm.edu.my; \\ arazman70@kedah.uitm.edu.my
}

\begin{abstract}
Edge detection is important in image analysis to form the shape of an object. Edge is the boundary between different textures, which helps with object segmentation and recognition. Currently, several edge detection techniques are able to identify objects but are unable to localize the shape of an object. To address this problem, this paper proposes a fusion of selected edge detection algorithms with mathematical morphology to enhance the ability to detect the object shape boundary. Edge detection algorithm is used to simplify image data by minimizing the amount of pixel to be processed, whereas the mathematical morphology is used for smoothing effects and localizing the object shape using mathematical theory sets. The discussion section focuses on the improved edge map and boundary morphology (EmaBm) algorithm as a new technique for shape boundary recognition. A comparative analysis of various edge detection algorithms is presented. It reveals that the LoG's edge detection embedded in EmaBM algorithm performs better than the other edge detection algorithms for fruit shape boundary recognition. Implementation of the proposed method shows that it is robust and applicable for various kind of fruit images and is more accurate than the existing edge detection algorithms.
\end{abstract}

Keywords: Image processing, edge detection, mathematical morphology, edge map, shape boundary recognition, mango images. 


\section{INTRODUCTION}

Edge detection is a fundamental process in image processing and it is the foundation for pattern recognition. Edge detection refers to the process of identifying and locating sharp discontinuities in an image (Maini \& Aggarwal, 2009; Sharma, Singh, \& Kaur, 2013). Discontinuities refer to rapid changes in pixel intensity which characterize different boundary regions, shadow boundaries and abrupt changes in surface orientation. Shape boundary detection is mainly used to detect the outline or shape of the object; thus, it can easily identify objects based on the shape. However, conventional edge detection algorithms usually generate edges that are not closed contours, which can lead to difficulties in recognizing objects (Pande Ankita \& Shandilya, 2013). The shape boundary detection is more challenging due to the uneven illumination of the images.

There are many kinds of algorithms for image edge detection. These algorithms are Roberts (Roberts, 1965); Prewitt (Prewitt, 1970); Sobel (Sobel, 1990); Laplacian of Guassian (LoG) (Marr \& Hildreth, 1980); and Canny's (Canny, 1986) algorithms. The main function of these algorithms is highpass filter with each of them having different characteristics and applicability. Edge detectors are the algorithms which filter out the useless information and preserve the useful information in image (Juneja \& Sandhu, 2013). These algorithms are involved in orientation; hence, anti-noise performance is generally poor (Caixia, Yu, Hui, \& Yao, 2014); and it is unable to extract the edges in dark images, dimmed images or noisy images (Maini \& Aggarwal, 2009). Mathematical morphology provides an approach to the processing of digital images for extracting the corresponding shape boundary in the image. Mathematical morphology uses the shape and structures of the image as the study objects. Mathematical Morphology is non-linear image processing which has advantages in image edge detection and could make the accuracy of edge detection accurate and remove noise for addressing characteristics of shapes (Caixia et al., 2014).

In this study, edge detection algorithms are used to extract the significant properties of objects in an image. This study fuses selected edge detection techniques with mathematical morphology to produce solid fruit images. Mathematical morphology is used for smoothing effects and localizing the shape of an object using mathematical theory sets. The objective of this study is to identify the best edge detector that can be integrated with mathematical morphology for better shape boundary recognition. The fusion of edge detector and mathematical morphology is used to generate edge map and boundary morphology $(\mathrm{EmaBm})$ algorithm. The results show that extracting the shape 
boundary using EmaBm has the advantage of producing higher accuracy shape boundary.

\section{METHODOLOGY}

Computer vision process consists of image acquisition, image preprocessing, segmentation, feature extraction and classification (Sharifah Lailee, Hamirul' Aini, Khudzir \& Nursuriati et al., 2010). However, the focus of this paper is on the segmentation technique which uses edge-based method and mathematical morphology. Segmentation process is crucial because the quality of segmented image affects the results of feature extraction and classification task (Shaharanee \& Jastini, 2015). The extraction task transforms sufficient content of images into various content features or attributes. In order to test the applicability of the proposed fusion method, the Harumanis mango fruit images are chosen to further illustrate the shape boundary recognition.

\section{EmaBm Algorithm}

The fusion of edge detection algorithm and mathematical morphology consists of edge map and boundary morphology (EmaBm). The EmaBm Algorithm is as follows:

EmaBm Algorithm

Input: $R G B$ Image

Output: Shape boundary

Begin

1. Input image, $I$

2. Do convertion $I$ to grayscale image, $I_{G}$ $I_{G} \leftarrow$ converttogray $(I)$

3. Do filtering $I_{G}$ using edge detection and generate edge map, $I_{E}$ $I_{E} \leftarrow \operatorname{edgemap}\left(I_{G}\right)$

4. Do cleaning $I_{E}$ by area opening and get edge simplification, $b_{1}$ $b_{1} \leftarrow I_{E}^{\circ}$ ao(50)

5. Do smoothing $b_{1}$ by series of morphological operations and get shape boundary, $b_{4}$

$b_{2} \leftarrow b_{1} \cdot \mathrm{S}_{\mathrm{E}}(\operatorname{disk}, 8)$

$b_{3} \leftarrow$ holefill $\left(b_{2}\right.$, holes $)$

End

$b_{4} \leftarrow b_{3} \Theta \mathrm{S}_{\mathrm{E}}($ disk, 8$)$ 


\section{Image Acquisition}

Samples of 120 Harumanis images were acquired from Bukit Bintang Agriculture Centre. The fruits were graded manually by a human grader as soon as the fruits were collected. Unfortunately, the manual grading processes were based on subjective evaluation which was bound to have human error. Harumanis images were taken in the laboratory under uncontrolled lighting using digital camera. All images were saved in Joint Photographic Expert Group (JPEG) format and displayed in red, green and blue (RGB) format with resolution of 1624 X 2448 pixels.

\section{Image Preprocessing}

Image preprocessing involves conversion of RGB image into gray scale image. The conversion uses 'rgb2gray' function in MATLAB. RGB image is converted to gray-scale image as shown in Figure 1.

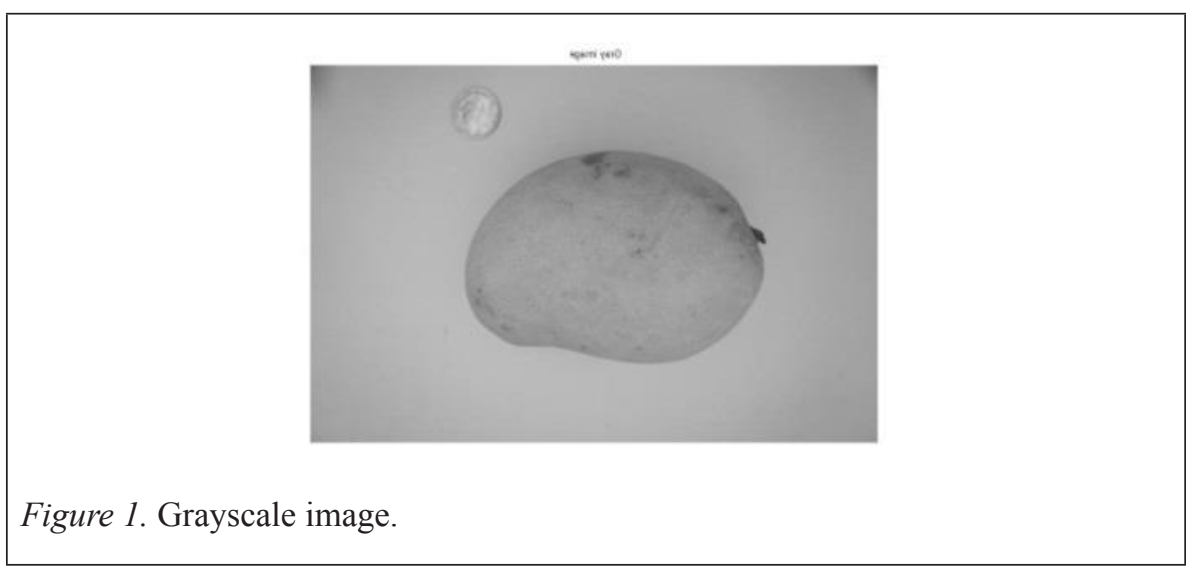

For an image $I$, the gray-scale image is expressed by:

$$
I_{G} \leftarrow \text { conver to gray }(I)
$$

where $I$ is the RGB image and $I_{G}$ is the gray-scale image. In gray scale image, the background tends to merge with the object being investigated. Therefore, image segmentation is performed to extract the object of interest from its background. Examples of the pixel intensity value for gray scale image are shown in Figure 2. 


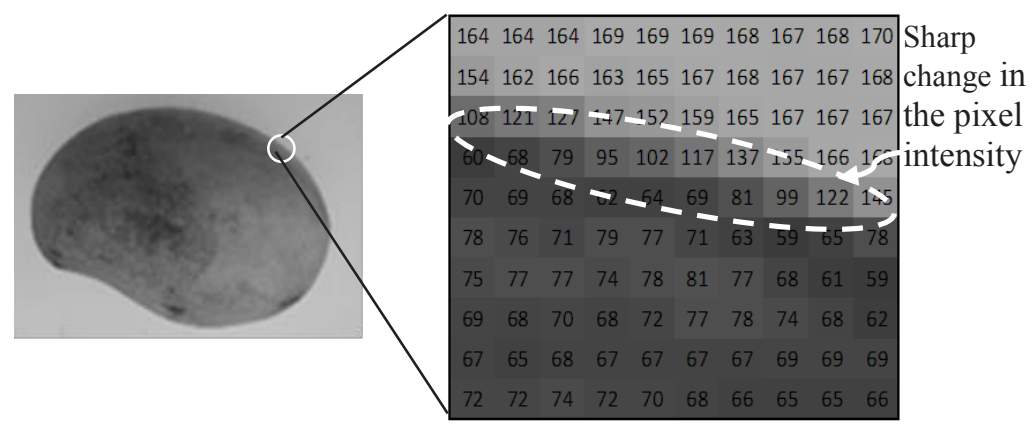

Figure 2. Pixel intensity for grayscale image, $I_{G}$

\section{Edge Map}

Edge map refers to the information about the position, strength, and orientation of edges. The edge map is derived by convolving gray-scale images and edge detection algorithm; data volumes in the image are significantly reduced without affecting the structural properties. The edge map $I_{E}$ is expressed by:

$$
I E \leftarrow \text { edge } \operatorname{map}(I G)
$$

Edge detection algorithm reads each pixel in a gray-scale image $I_{G}$, then changes the pixel intensity to 1 (white) or 0 (black). If a significant change occurs at a given pixel, a white pixel is placed in the edge map; otherwise, a black pixel is placed instead. Figure 4 shows an example of pixel intensity for gray-scale image $I_{G}$ which has been converted to pixel intensity of edge map $I_{E}$.

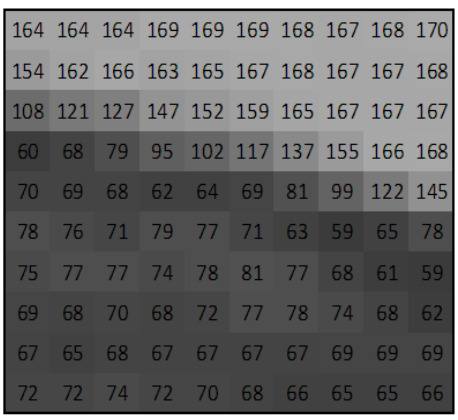

\begin{tabular}{|llllllllll|}
0 & 0 & 0 & 0 & 0 & 0 & 0 & 0 & 0 & 0 \\
\hline 1 & 0 & 0 & 0 & 0 & 0 & 0 & 0 & 0 & 0 \\
1 & 1 & 1 & 1 & 1 & 0 & 0 & 0 & 0 & 0 \\
0 & 0 & 0 & 0 & 1 & 1 & 1 & 1 & 0 & 0 \\
\hline 1 & 0 & 0 & 0 & 0 & 0 & 0 & 1 & 1 & 1 \\
\hline 0 & 0 & 0 & 0 & 0 & 0 & 0 & 0 & 0 & 0 \\
\hline 1 & 1 & 1 & 1 & 0 & 0 & 0 & 0 & 0 & 0 \\
\hline 0 & 0 & 0 & 0 & 1 & 1 & 1 & 0 & 0 & 1 \\
\hline 1 & 1 & 1 & 0 & 0 & 0 & 0 & 0 & 0 & 0 \\
\hline 0 & 0 & 0 & 0 & 0 & 0 & 0 & 0 & 0 & 0 \\
\hline
\end{tabular}

Figure 4. Pixel data for grayscale image, $I_{G} \rightarrow$ edge map, $I_{E}$ 
Examples of the edge maps $I_{E}$ represented by logical matrix are shown in Figure 5.1

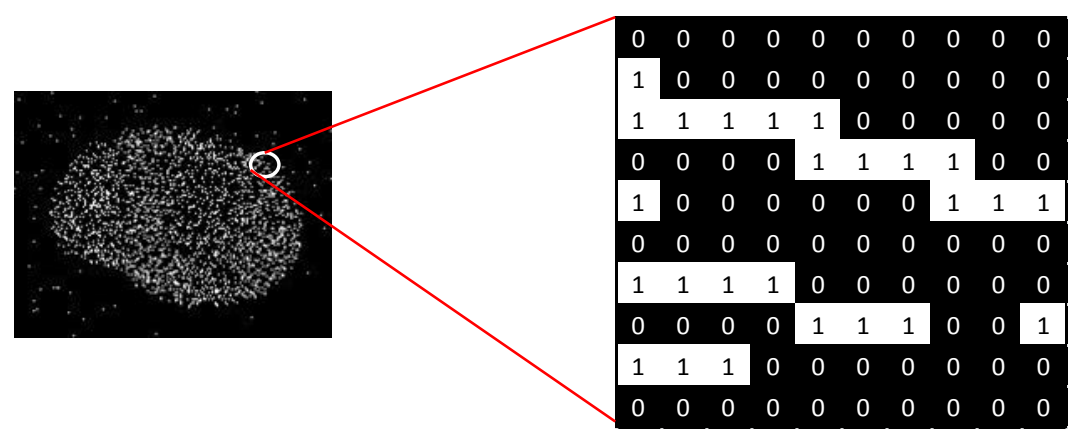

Figure 5. Edge map $I_{E}$ represented by logical matrix.

Edge maps are edges that are not connected and appear as micro-pseudo edges with discontinued edge segments, thus degrading the accuracy of location and isolating edge points. Therefore, these edge points are usually discarded in the boundary morphology phase.

\section{Boundary Morphology}

The second phase which is the boundary morphology phase consists of two steps:

Edge Simplification. In edge simplification, pixels of object in an image are cleaned while maintaining their edge characteristics. The cleaning process is done by an area opening operator, that removes the connected components which have areas that are smaller than a predefined number of pixels. In this study, the predefined pixel is 50. During this process, an area opening operator is applied to edge map $I_{E}$ using the 50 pixels. This operator removes all objects which are smaller than 50 pixels from edge map $I_{E}$. This operation cleans up unnecessary edge points but maintains pixels that are part of the objects. The edge simplification of $I_{E}$ with respect to area opening $a o$ is expressed by:

$$
b 1 \leftarrow I E \circ a o(50)
$$

Where $a o$ is the number of pixels and $b_{1}$ is the simplified edges. The result of edge simplification, $b_{1}$ is shown in Figure 6. 


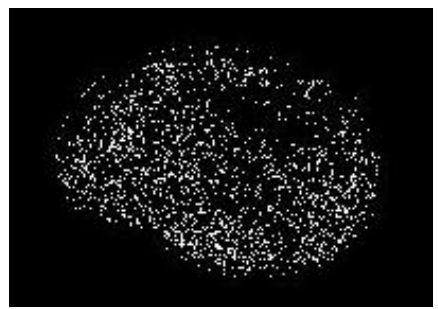

Figure 6. Edge simplification, $b_{1}$

Shape morphology. Shape morphology refers to noise smoothing process using multiple morphology operators to preserve the shape of an object. This process uses a closing operator to smooth the edges of an object and eliminate false touching in order to obtain the shape of the object. A closing operator is applied to $b$, using a disk-shaped structuring element with a radius of eight pixels. The disk-shaped structuring element is used to preserve the circular nature of the object and the radius of eight pixels is used to fill the largest gap in the object. The closing operator preserves foreground regions, while eliminating other regions of background pixels to obtain a coarse object shape. The closing operation on $b_{1}$ with respect to structuring element $S_{E}$ is expressed by:

$$
b 2 \leftarrow b 1 \cdot S E(\operatorname{dis} k, 8)
$$

where $S_{E}$ is the structuring element and $b_{2}$ is the coarse object shape. The result of coarse shape, $b_{2}$ is shown in Figure 7 .

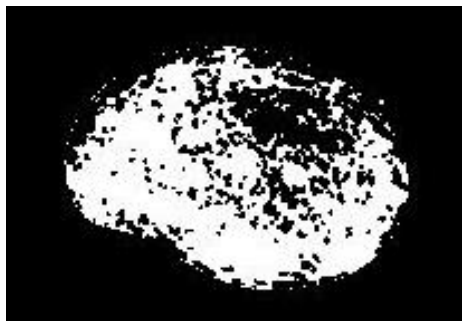

Figure 7. Coarse object shape, $b_{2}$

Image morphological closing operation only fills small gaps in image components. Therefore, the hole filling morphological operation is required 
to preserve the shape by changing the intensity of regions that are marked as holes in the foreground intensity. Hole filling operation on $b_{2}$ is expressed by:

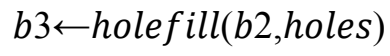

where holes is the mask to fill the object and $b_{3}$ is the object shape. The result of $b_{3}$ is illustrated in Figure 8.

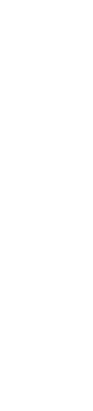

.

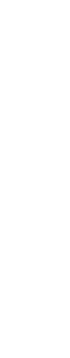

כ

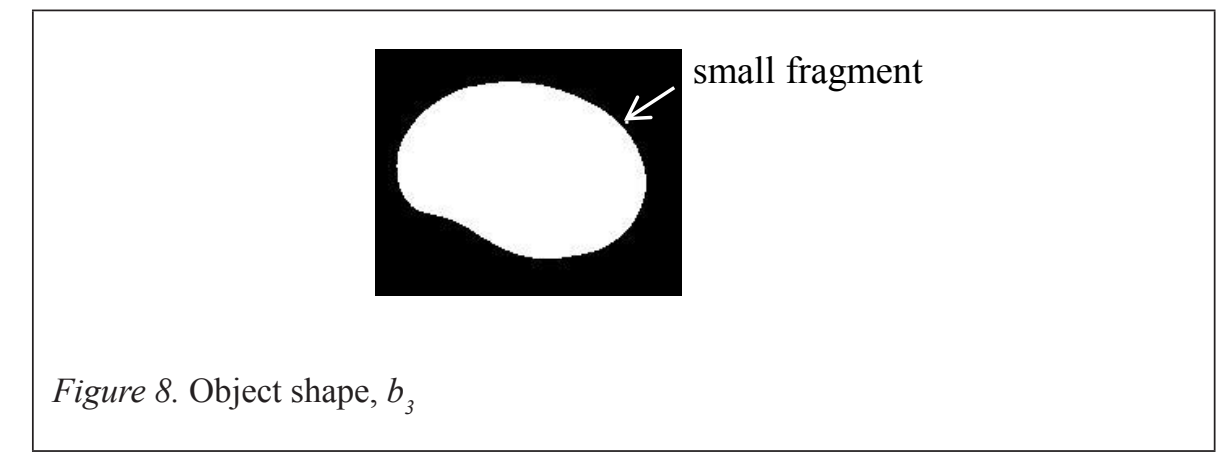

After identifying the object shape, it is important to properly extract the shape. However, due to the weak edges of the images, some small fragments of non-objects with similar intensities are also connected to the object. These fragments must be removed. To remove these fragment, an erosion operator is applied to $b_{3}$ using a disk-shaped structuring element $S_{E}$ with a radius of eight pixels. This process separates non-objects from the object. Erosion operation on $b_{3}$ with respect to $S_{E}$ is expressed by:

$$
b 4 \leftarrow b 3 \ominus S E(\operatorname{disk}, 8)
$$

where $b_{4}$ is the shape boundary. Figure 9 illustrates the result after performing EmaBm algorithm.

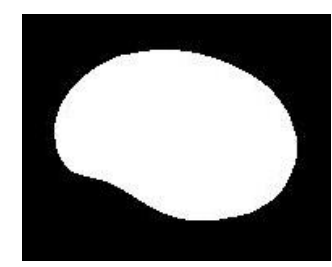

Figure 9. Shape boundary, $b_{4}$ 


\section{RESULT AND DISCUSSION}

The experiments were carried out to evaluate the shape boundary result of fusing the various edge detection algorithms with mathematical morphology. Table 1 illustrates the performance of various edge detection algorithms embedded in the EmaBm algorithm.

Table 1

Shape boundary of Harumanis image using various edge detection algorithms embedded in EmaBm algorithm

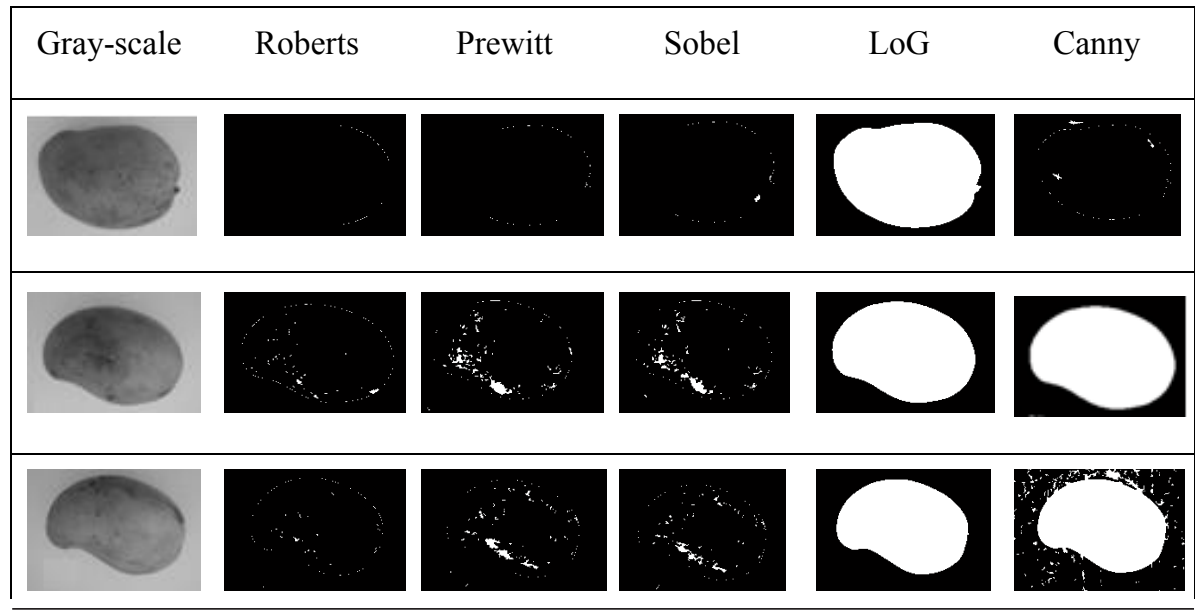

The result shows that Roberts, Prewitt and Sobel's algorithms provide low quality edge maps relative to the others. This is because these algorithms use fixed kernel filter size and coefficients which cannot detect specific edges for Harumanis images. These algorithms produce missing and discontinuous edges of the object because the fixed kernel filter is only able to track horizontal and vertical pixels.

Canny algorithm is able to detect specific edge for Harumanis images. However, Canny algorithm still suffers from mismapping of some of the edges. Nevertheless, in some images, Canny algorithm is able to detect edges of the images. This is because Canny algorithm has multiple filtering kernel which enables this algorithm to detect both strong and weak edges.

Lastly, this study shows that LoG is the best algorithm embedded in EmaBm because this algorithm is able to detect the edge for all 120 of Harumanis images. LoG algorithm is able to detect both strong and weak edges in various 
orientations, smooth the images and locate the correct places of edges to avoid detection of false noise components in the images. Therefore, it can be concluded that LoG edge detection algorithm exhibits good performance with EmaBm in extracting Harumanis shape boundary. To verify this result, the image of papaya and carrot were extracted using LoG with EmaBm. This newly improved algorithm was able to extract the shape of these fruits without giving false noise components of the images as shown in Table 2. Therefore, it can be concluded that this improved algorithm is superior for detecting the shape boundary of fruits.

Table 2

The EmaBm Algorithm on Different Fruit Images

\begin{tabular}{|c|c|c|}
\hline Fruit & Gray-scale & LoG \\
\hline Papaya & & \\
\hline Carrot & & \\
\hline
\end{tabular}

\section{CONCLUSION}

Shape boundary detection is a challenging process due to the cluttered image, non-uniform illumination and complex background. Using edge detection algorithm only is not sufficient to detect the outline of an object. Therefore, an improved fusion of edge detection algorithm with mathematical morphology, EmaBm was developed to enhance the ability of extracting the shape boundary of fruit images. In this study, existing edge detectors algorithm were tested for compatibility with EmaBm. Results show that only LoG algorithm with EmaBm is able to randomly detect the shape boundary for all Harumanis mango fruit images, and seems to be more suitable than the other edge detection algorithms. 


\section{REFERENCES}

Caixia, D., Yu, C., Hui, B., \& Yao, H. (2014). The improved algorithm of edge detection based on mathematics morphology. International Journal of Signal Processing, Image Processing and Pattern Recognition, 7(5), 309-322. doi:http://dx.doi.org/10.14257/ijsip.2014.7.5.27

Canny, J. (1986). A computational approach to edge detection. IEEE Transactions on Pattern Analysis and Machine Intelligence, 8(6), 67998. Retrieved from http://www.ncbi.nlm.nih.gov/pubmed/21869365

Juneja, M., \& Sandhu, P. S. (2013). An analysis of edge detectors and improved hybrid technique for edge detection of images. International Journal of Computer Science and Electronics Engineering, 1(4), 469-477. Retrieved from http://www.isaet.org/images/extraimages/L813086.pdf

Maini, R., \& Aggarwal, H. (2009). Study and comparison of various image edge detection techniques. International Journal of Image Processing, 3(1), 1-12. Retrieved from http://www.math.tau.ac.il/ turkel/notes/ Maini.pdf

Marr, D., \& Hildreth, E. (1980). Theory of edge detection. Proceedings of the Royal Society of London B: Biological Sciences, 207(1167), 187-217.

Pande Ankita, V., \& Shandilya, V. K. (2013). Digital image processing approach for fruit and flower leaf identification and recognition. International Journal of Engineering and Computer Science, 2(4), 1280-1285. Retrieved from ijecs.in/ijecsissue/wp-content/uploads/2013/04/12801285ijecs.pdf

Prewitt, J. M. (1970). Object enhancement and extraction. Picture Processing and Psychopictorics, 10(1), 15-19.

Roberts, L. (1965). Machine perception of three-dimensional solids. MIT Lincoln Laboratory Technical Report, 159-197.

Shaharanee, I. N. M., \& Jastini, M. J. (2015). Irrelevant feature and rule removal for structural associative classification. Journal of Information and Communication Technology, 14, 95-110. Retrieved from http:// www.jict.uum.edu.my/images/pdf/vol14/jict146.pdf 
Sharifah Lailee, S. A., Hamirul'Aini, H., Khudzir, I., \& Nursuriati, J. (2010). Improved technique for segmenting images under natural environment. International Conference on Science and Social Research, 401-405. doi:10.1109/CSSR.2010.5773808

Sharma, P., Singh, G., \& Kaur, A. (2013). Different techniques of edge detection in digital image processing. International Journal of Engineering Research and Applications, 3(3), 458-461. Retrieved from http://www. ijera.com/papers/Vol3_issue3/BY33458461.pdf

Sobel, I. (1990). An isotropic 3× 3 image gradient operator. In Machine Vision for Three-Dimensional Sciences (pp. 376-379). New York: Academic Press. 